\title{
An Experience on the Correlation Analysis Between Academic Marks and Emotions
}

\author{
http://dx.doi.org/10.3991/ijet.v9i7.3702 \\ Ruth Cobos, Francisco Jurado, Álvaro Ortigosa, Pilar Rodríguez \\ Universidad Autónoma de Madrid, Madrid, Spain
}

\begin{abstract}
Observe appropriate evidence that pointing emotions plays an important role in the learning process. However, there is no precedent of a research analyzing the relationship between emotions and academic marks using text analysis. Thus, in this article, we show the experience we have performed in order to analyze the possible existing correlations between student marks, assigned by both their classmates and by their instructors, and the emotion traces that can be found in their writings. To that end, we gathered data corresponding to text contributions of a course on Computer Systems in our University and perform the correspondent analysis. The obtained results look to indicate that some kind of correlation exists between marks and emotions in both the highest and the lowest marks.
\end{abstract}

Index Terms-Computer Supported Collaborative Learning (CSCL); Affective Computing for Learning; e-Assessment.

\section{INTRODUCTION}

There are clues spotting out that emotions play an important role in the learning process and that the influence of emotions on that process can be pointed out by observing the right evidences. In this regard Beard [1] states that student emotions have a significant effect on their engagement in the learning process. Besides, Suzuki et al. [2] found evidence that emotional clues while reading an article, prompt a learner to write an essay to engage in it, and also result in the learner to better use her/his knowledge to argue the issues. That is, students using an emotional tag set for annotating documents had better results that those using a rational tag set.

In order to identify the emotional evidences, there are several emotion measurement models, methods and tools in educational settings, which can be grouped into three wide areas [3]: Psychological, which uses verbal and nonverbal self-reporting of emotions; Physiological, which makes use of sensors in order to measure people's physiological reactions; and Motor-Behavioral, which measures behavioral expressions and changes in physical body that communicate one's emotion experience.

Under this classification, there are works that acknowledge the importance of emotion identification in the learning context by proposing mechanisms for leveraging those emotions. For instance, in [4] and [5] we can find mechanisms for detecting student emotional states from voice analysis of students' interactions within an elearning system. Also with the aim of integrating emotions identification into an Intelligent Tutoring System, Xiaoqing et al. [6] propose to extend the recognition mechanism by adding heterogeneous sensory data (e.g. speech and visual appearance).
However, the main drawback of these techniques is the amount of necessary widgets and sensors in order to collect the data to perform emotion detection. This situation leads us to think that emotion detection in texts is one of the least invasive measurement procedures. Natural language does not only transmit information, but also emotions and affects. Both goals do not necessarily are intended at the same time: while different literary genre would stimulate different emotional states in the reader, textbooks aim to be essentially informative. However, even when writing in a formal context, documents can express different emotions.

This leads to a specific topic from the Natural Language Processing and called sentiment analysis or opinion mining. Feldman defined it as the task of finding the opinions of authors about specific entities [7]. Another extended and accepted definition is the one given by Liu, who stated that it is the computational study of sentiments in text [8]. In this research, we have followed this last approach.

Therefore, the final goal of emotion detection in text is to identify what emotions can be found in any kind of text, not limited to self-reports on the subject own feelings. In this sense, there are different research works, systems and applications available, specifically centered in analyzing individual short texts. Current researches show how sentiment analysis has been applied to many different contexts like marketing, politics, shopping, etc. [7]. In addition, we can highlight the work presented by Strappavana and Mihalcea [9], which shows several experiments concerned with the emotion analysis of news headlines, and proposes and evaluates some methods to identify emotions in that kind of short texts. In this regard, Tian et al. [10] proposes to recognize emotions from interactive (Chinese) texts [11] by using techniques such as data mining for pattern extraction of e-Learner emotion communication.

In this direction, we have carried out some studies with the goal of assessing the effect of emotions in student motivation [12][13]. In those studies we have used a word-spotting technique to detect emotions and, possible, motivation in academic essays. Preliminary results shows signs of correlation between motivation and the emotions conveyed by the essays.

Thus, in this context, as far as we know, there is no precedent of a research analyzing the relationship between emotions and academic marks using text analysis. For this reason, in this article we will show the research study we have performed analyzing the essays written by students and assessed both by student peers and by the course instructors. The goal of the research is to verify whether there exists any relation between the work marks and the 
emotions transmitted by the text of the essays. To do so, our goal was to study whether any correlation existed between the data available: instructors' marks, classmates' marks and identified emotions.

Along the article, we will detail the experimentation carried out, in which we collected data corresponding to an experience that took place during an academic year on a course on Computer Systems at our university. That course was part of the syllabus of the Computer Science and Engineering studies. In that experience, each student was supposed to contribute by writing short technical documents about course topics. Their contributions were qualified both by their classmates and their instructors. These contributions in form of documents and the classmates' marks were supported by the CSCL system called KnowCat [14][15][16]. In the experience, we selected a set of contributions, whose marks were available, both by colleague students and instructors. Then, using a wordspotting technique for emotion analysis, we examined those contributions, finding which emotions could be identified in them.

This paper is organized as follows. Firstly, Section II introduces the environment we used as starting point in our research work. Then, Section III presents the plan of the experience, exposing the goal defined, the participants involved, the environment they used, the input data and variables to analyze, the how experience itself was performed and the result analysis we obtained. Finally, Section IV closes with some concluding remarks and future work.

\section{CONTEXT AND USED ENVIRONMENT}

In order to carry out the experiment, our starting point was the CSCL system called KnowCat (acronym for "Knowledge Catalyser") [14][15][16]. This is a fully consolidated and thoroughly tested and validated CSCL system that is in action since 1998. The main aim of this system is to generate quality educational materials as an automatic result of student interactions with the learning materials, by catalyzing the crystallization of knowledge [14][15][16].

Figure 1 illustrates an example of the "Computer Systems" KnowCat site. As we can see, it enables us to build up community knowledge sites -or knowledge sites for short-, where the educational materials are organized in a knowledge tree, which is a hierarchy of topics (area A). Each topic contains a set of mutually alternative documents that describe the topic. At any given time, all documents contained in the same topic compete with each other to be considered as the "best" description of the topic. This competitive environment is achieved by the Knowledge Crystallization mechanism of the system.

In short, Knowledge Crystallization is a process where the opinions of other users about a knowledge element (i.e. a document or an essay) and the evolution these opinions received, determine what knowledge elements are socially accepted. In such case, these elements of knowledge remain longer in the system; otherwise, if they turn out to be useless, they end up disappearing from the system.

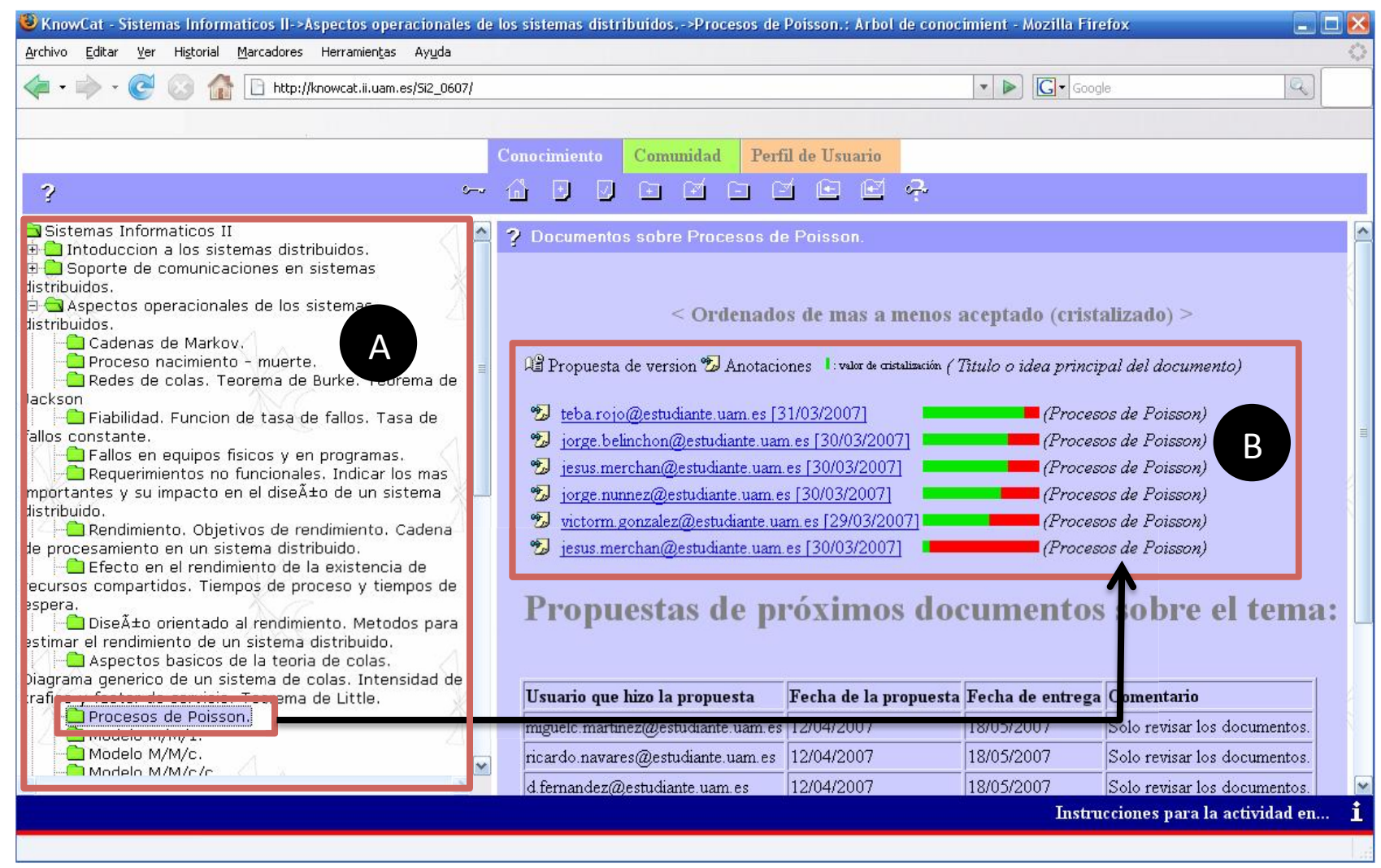

Figure 1. Screenshot of the KnowCat tool. 


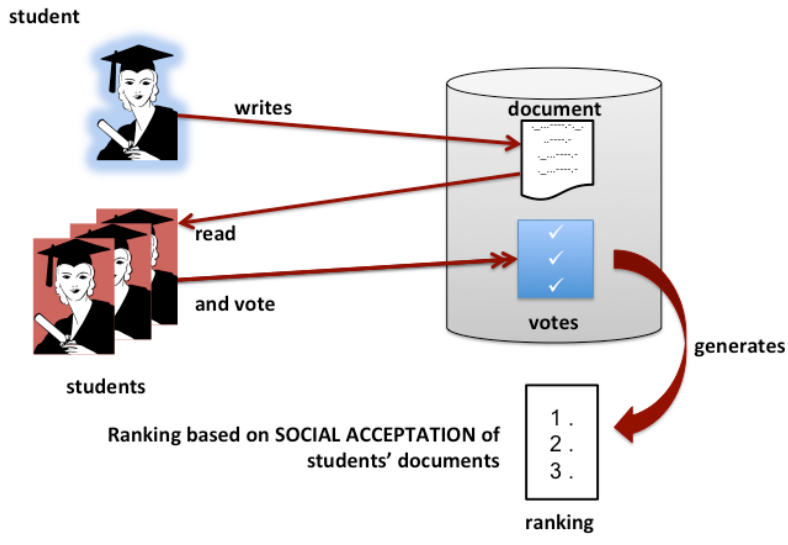

Figure 2. Social acceptation calculation in KnowCat.

Hence, students can collaborate in the system by adding documents to the knowledge site topics, voting documents, annotating documents and adding new document versions.

Back to figure 1, the B area shows how documents are identified by the author's e-mail address, arrival date and title. They are ordered by their degree of social acceptance, which is shown to the right of the identification heading of each document with the green-red bar. On the left side of the identification heading of each document, the icons indicate whether a document has received annotations and whether a new version of the document is available. As an example, the document identified by "teba.rojo@estudiante.uam.es [31/03/2007]" (the first element of the rank) shows the highest degree of acceptance in the topic called "Procesos de Poseidon" (Poseidon's Processes).

In addition, we have integrated to the KnowCat system a tool that uses the word-spotting technique in order to analyze the emotional clues. This technique for emotional identification in texts is based on associating a set of emotion evocative words to each of the four emotions we center on, namely Joy, Sadness, Fear and Anger. That is, a set of related words was built using a cross-linguistic approach. Then, if any of the words of the set (or some or its derived words) are found in the text, it increases the corresponding emotional load (more information can be found at [12] and [13]).

Therefore, as we can see, the KnowCat tool provides the necessary settings that allow us to perform the experience within the context of a CSCL environment that uses the word-spotting technique to detect emotions in academic essays. Next, we will detail the plan of the experience.

\section{EXPERIENCE'S PLAN}

In this section we will present in detail the plan of the experience. Thus, along the different subsections we will define the goal, present the implicated participants, expose the input data and variables to analyze, describe how experience itself was performed and finally analyze the obtained results.

\section{A. Goal Definition, Participants and Their Role-part}

According to the research motivation we set in the introduction section, we defined the goal of our experience as follows: analyze instructors' marks, classmates' marks and identified emotions for the purpose of identify if any correlation exists among them.
In the conducted experience, a group of 160 students of the course "Computer Systems" in Computer Science and Engineering studies from our University of Madrid (in Spanish Universidad Autónoma de Madrid) was involved during the academic year 2006/2007.

That course was part of the syllabus of the Computer Science and Engineering studies. From the 160 students enrolled in that course, 100 students participated actively and accomplished their tasks in the experience.

Each student was supposed to contribute by writing short technical documents about the enrolled course topics. Afterward, the contributions were qualified both by their classmates and their instructors. More discussion on the scientific methodology that was adopted will be explained better in the experience process definition subsection.

\section{B. Input Data and Variables Definition}

For the present research work, we collected 168 contributions whose marks were available both students and instructors. The requirements for them to be selected were having their both marks available. That way, classmates and instructors were the same in all cases and document emotions would affect their marks in the same way, if any.

Then, using a word-spotting technique for emotion analysis, we examined those contributions, finding which emotions could be identified in them.

In this research study, for each student contribution (i.e. technical document) we have collected the following data: i) its social acceptation, which is calculated through the classmates' marks; ii) its objective quality measure, which is calculated through the instructors' marks and iii) its emotion traces.

Firstly, the KnowCat provided the social acceptation of a document as shown in figure 2. In that figure we can see how this social acceptation is calculated by the KnowCat knowledge crystallization mechanism explained in the previous section. It takes into account the students' marks in form of votes for each specific document. That is, this value is a measure of the document quality in the collaborative opinion of the students. Its value is into the range $[0,1]$.

Secondly, the objective quality measure of a document was the arithmetical mean among their instructors' marks. It is a value into the range $[0,10]$.

Thirdly, each document was analyzed using an emotion extraction tool [12][17]. The tool, based on a wordspotting technique, supports the evaluation of the emotional classification proposed by Zing \& Newen [18] conveyed by a given piece of text, namely: joy, sadness, anger and fear. The identification of those four emotions as the basic ones is based on [18]. Each emotion data is a value into the range $[0,1]$. The current version of the tool works with documents in Spanish.

To clarify our explanation, table 1 shows an example with the assigned value for a set of student contributions to the above-mentioned data, which are in the first column. The second column contains values for a document, which is qualified with good quality by instructors and students. However, the fourth columns have values for one document, which is qualified with no good quality by instructors and students. 
TABLE I.

DATASET EXAMPLE FOR THE RESEARCH STUDY

\begin{tabular}{|c|c|c|c|c|c|}
\hline \multicolumn{2}{|c|}{ Contribution Identification } & 17 & 18 & 45 & $\ldots$ \\
\hline \multicolumn{2}{|c|}{ Author Identification } & 13 & 22 & 19 & \\
\hline \multicolumn{2}{|c|}{$\begin{array}{l}\text { Classmates' Mark } \\
\text { (Social Acceptance) }\end{array}$} & 0,99 & 0,50 & 0,35 & \\
\hline \multicolumn{2}{|c|}{$\begin{array}{l}\text { Instructors' Mark } \\
\text { (Quality Measure) }\end{array}$} & 8 & 6,7 & 4 & \\
\hline \multirow{5}{*}{ 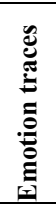 } & \% Joy & 0,0073 & 0,0007 & 0,0007 & \\
\hline & \% Sadness & 0,0012 & 0,0007 & 0,0007 & \\
\hline & $\%$ Anger & 0 & 0,0007 & 0,0024 & \\
\hline & $\%$ Fear & 0,0110 & 0,0063 & 0,0015 & . \\
\hline & $\Sigma \%$ emotions & 0,0196 & 0.0087 & 0,0055 & \\
\hline
\end{tabular}

\section{Experience Process Description}

Taking into account the goal defined, the participants involved, the environment they used, the input data and variables to analyze, in this subsection we will describe the experience we performed following the diagram shown in figure 3 , which we will use in order to conduct our explanation.

Thus, on the left and center of figure 3 we can see how, as introduced above, each student had to produce a small technical document (fragment or description) on some assigned topics and vote with a value into de range $[0,10]$ for all the documents (of their classmates) in those topics. At the end of this experience, there were on average 5 documents per topic.

The knowledge site of this course started with a knowledge tree composed of approx. 60 topics (the course structure was created by the course instructors) and without any documents. The students worked on 40 of these 60 topics. The main topic of this course was "Distributed Systems". In this course, the students studied concepts related to performance, operational and communication aspects of distributed systems and Middleware.

At the end of this experience, as we can see on the right of figure 3, course instructors evaluated the students' work. They assigned a mark to each document with a value into the range $[0,10]$ taking into account its quality.

As a result, on the one hand, we obtained a ranking of the documents for each topic of the knowledge tree, taking into account the marks assigned to them by the instructors. On the other hand, another ranking was offered by the KnowCat system through its knowledge crystallization mechanism. Then, we analyzed all the selected documents from the emotion point of view (on the right-center of figure 3).

\section{Analisys and Interpretation}

For a given document, the final classmates' mark assigned is, somehow, related to its social acceptation; whilst the instructors' mark is accepted as being a more objective quality measure. Anyway, we had always looked at both marks as a result of the technical content of the document itself: the better content the higher mark.

Once we had the data available, in the bottom of figure 3 we can see how we started by performing the correlation analysis for all the variables we were interested in; i.e.: classmates' mark, instructors' mark, joy, sadness, anger, fear and the total emotion level.

However, we wondered whether it could be the case that the document itself contained other kind of information, specifically certain level of joy, sadness, anger and fear, which would also be related to the final marks.

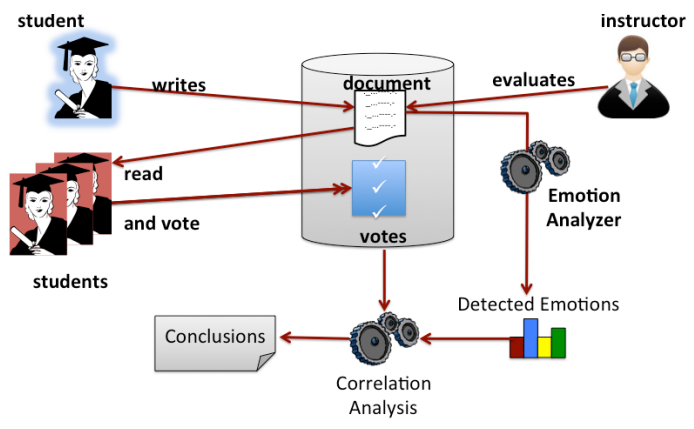

Figure 3. Experience process.

An example of the final data available is presented in the former Table I. The average number of words per document, arithmetic mean, was 874 (483 standard deviation), with a median of 834 .

With the data available, we started by calculating the Pearson correlation coefficient $r$ for all the variables we were interested in, namely: classmates' mark, instructors' mark, joy, sadness, anger, fear and the total emotion level. That "total emotion level" was obtained as the sum of the former four independent emotion levels. But there was no statistical significance in the correlation matrix that we obtained.

Then, we thought that it could be interesting to analyze different sections of the table independently. Specifically, we focused on different ranks of the two kind of marks assigned to each document (classmates' and instructors'). We were looking for the impact of emotions in the perceived quality of the documents, depending on their social acceptation and their objective quality measure.

As mentioned above, no significant correlation seemed to exist between both marks. The results we obtained in the analysis carried out offered interesting results in:

- the set of works with highest instructor marks.

- the set of works with lowest classmates' marks and those above the average.

Let's go into detail in the analysis in the subsections below.

As explained previously, instructors' marks range from 0 to 10 , and marks over 7 are considered to be high marks. The results we obtained in this rank are depicted in Tables II and III.

In Table II, we present the correlation results obtained for instructors' marks greater than or equals to $7.5(n=67$ in that rank). Those results suggest that a positive correlation exists between the social acceptation (classmates' marks) and the detected levels of joy and fear in the analyzed documents. In figure 4 we can observe this tendency graphically by using the diagram with the correlation coefficient for these data.

1) For instructors' highest marks: correlation between social acceptation and emotions

Furthermore, such correlation is stronger if we analyze the documents whose instructors' marks are greater than or equals to 8 . In that case, not only the $r$ values for joy and fear increase, but also a positive correlation exists regarding sadness and, also, the total amount of emotions detected in the documents, as presented in Table II. In this last case (instructors' marks $>=8$ ), the number of subjects, $n$ was 40 . Table III expose the related results and their corresponding correlation diagram is plotted in figure 5 . 

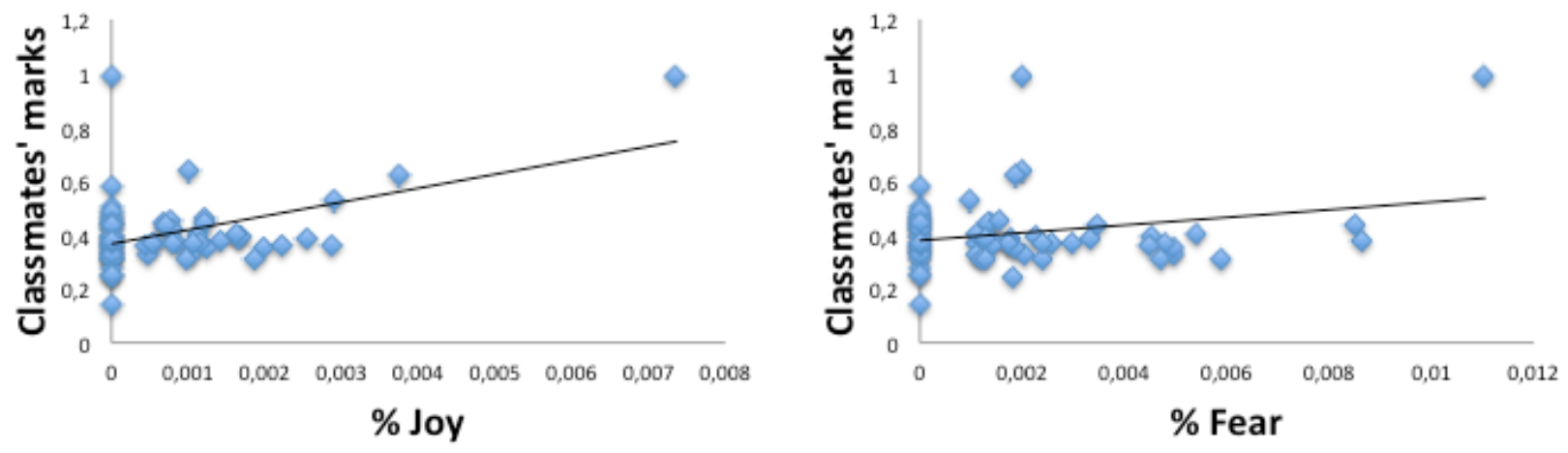

Figure 4. Diagrams with correlation coefficient between classmates' marks and emotions (joy and fear) for instructors' marks greater than or equals to 7.5

TABLE II.

FOR INSTRUCTORS' MARKS GRETTER THAN OR EQUALS TO 7.5: CORRELATION BETWEEN CLASSMATES' MARKS AND EMOTIONS $(N=67)$

\begin{tabular}{|l|l|l|}
\hline Variable & $\mathbf{r}$ & $\mathbf{p}$ \\
\hline \%Joy & 0,46538923 & 0,001 \\
\hline \%Fear & 0,25119097 & 0,05 \\
\hline
\end{tabular}

TABLE III.

FOR INSTRUCTORS' MARKS GRETTER THAN OR EQUALS TO 8: CORRELATION BETWEEN CLASSMATES' MARKS AND EMOTIONS $(N=40)$

\begin{tabular}{|l|l|l|}
\hline Variable & $\mathbf{r}$ & $\mathbf{p}$ \\
\hline \%Joy & 0,6713 & 0,001 \\
\hline \%Sadness & 0,4875 & 0,01 \\
\hline \%Fear & 0,4434 & 0,01 \\
\hline \%Emotions & 0,4916 & 0,01 \\
\hline
\end{tabular}
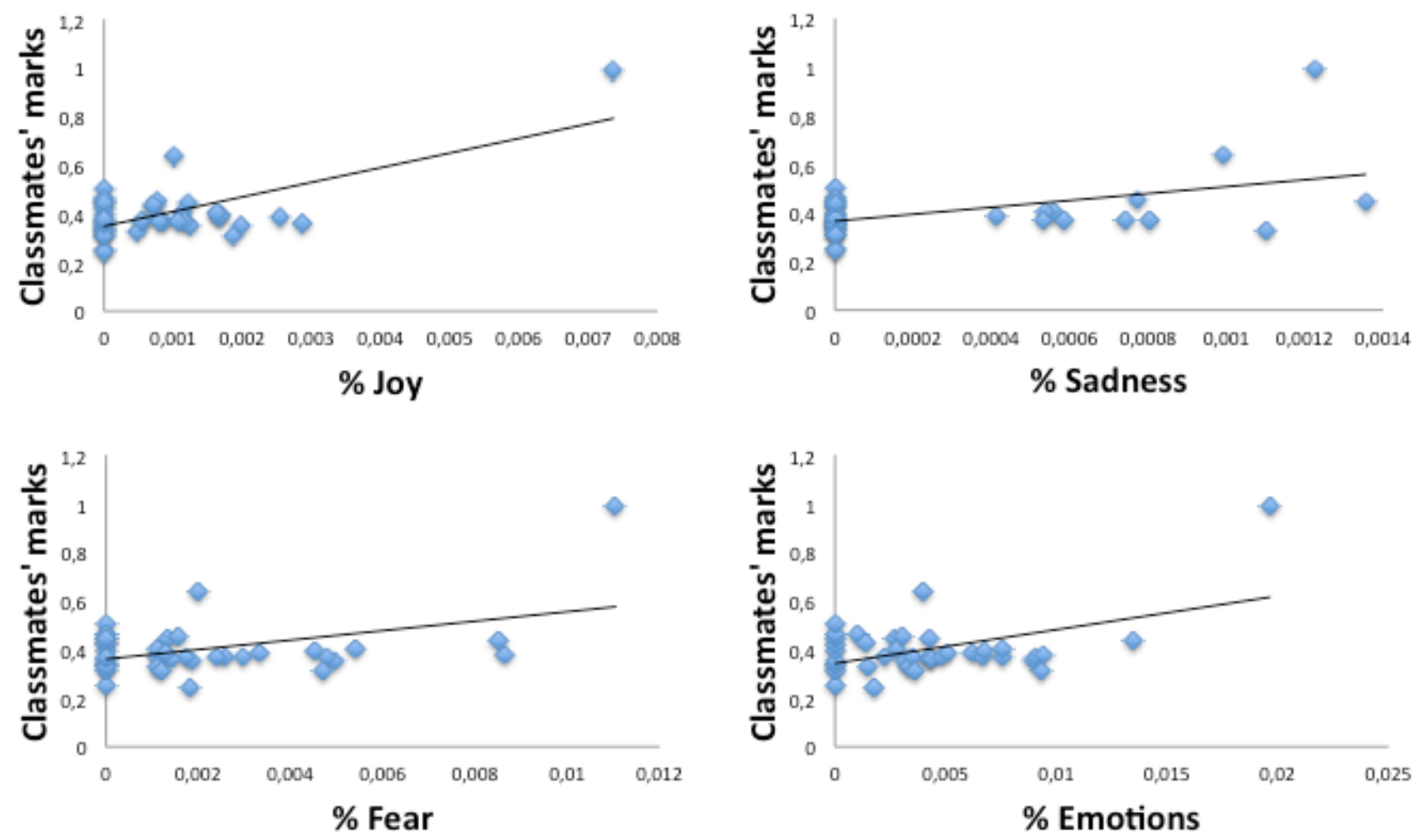

Figure 5. Diagrams with correlation coefficient between classmates' marks and emotions (joy, sadness, fear and the total emotion level) for instructors' marks greater than or equals to 8 

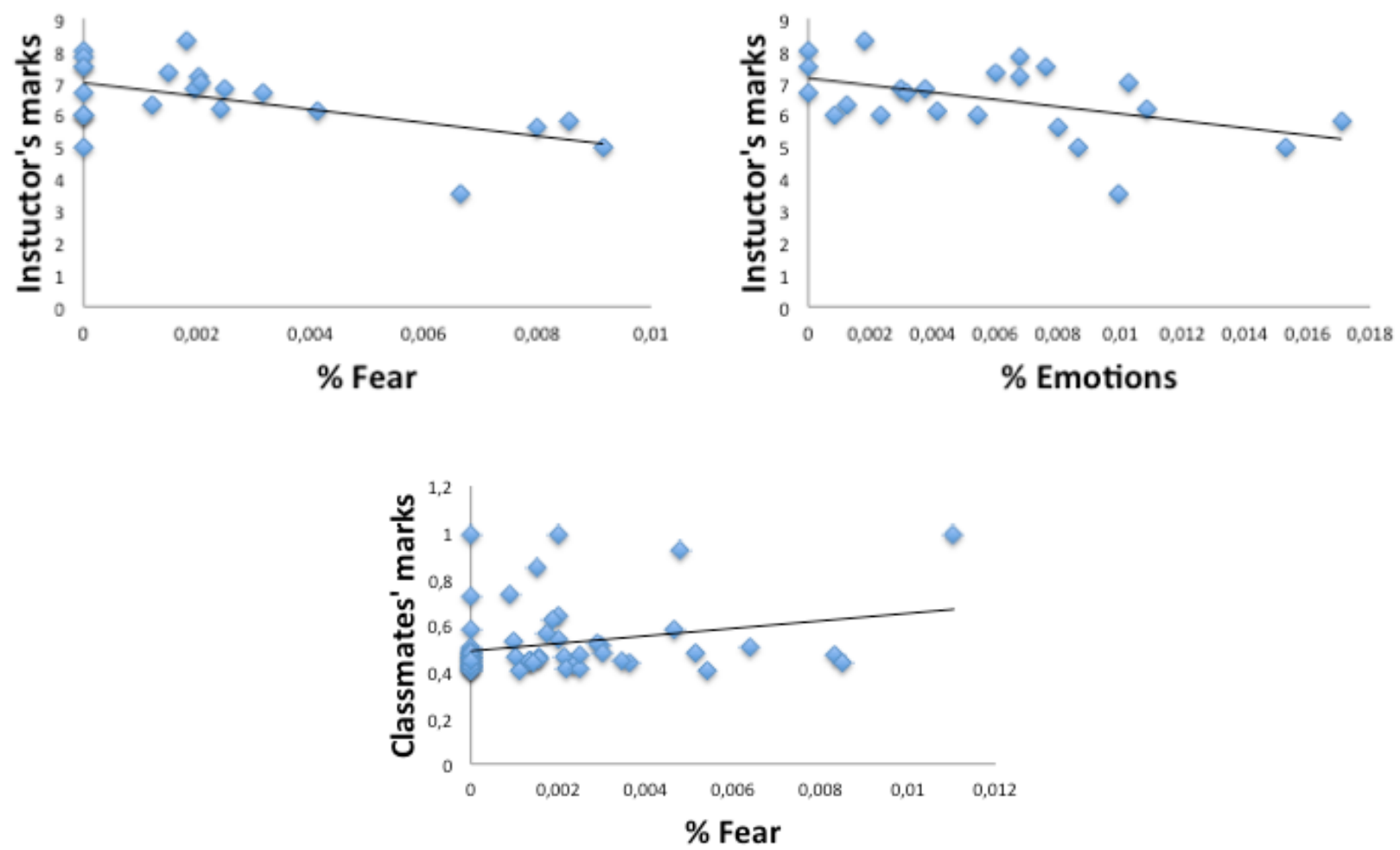

Figure 6. Diagrams with correlation coefficient between instructors' marks and emotions for classmates' marks lower than 0,3 (at top) and between classmates' marks and emotions for classmates' marks greater than or equals to 0,4 (at bottom)

2) For classmates' marks: correlation between objective qualifications and emotions

Classmates' marks range between 0 and 1; so, marks below 0.3 can be considered as being low marks. We can see the correlation diagram at the top part of figure 6 . In that analyzed range, we found out that a negative correlation exists between the instructors' marks assigned and the detected levels of fear and the total amount of emotions. The number of subjects $n$, in this analysis was 23 , Table $\mathrm{V}$ shows the $r$ and $p$ values from the Pearson correlation analysis.

TABLE IV.

FOR CLASSMATES' MARKS LOWER THAN 0,3: CORRELATION BETWEEN INSTRUTORS' MARKS AND EMOTIONS $(N=23)$

\begin{tabular}{|l|l|l|}
\hline Variable & $\mathbf{r}$ & $\mathbf{p}$ \\
\hline \%Fear & $-0,55499611$ & 0,01 \\
\hline \%Emotions & $-0,47822103$ & 0,05 \\
\hline
\end{tabular}

Moreover, if we look at the classmates' marks above the average at the bottom part of figure 6 (in this case, arithmetic mean was 0.3995 , with SD of 0.1297 and a median of 0,3710 ), we get a positive correlation between classmates' marks and the level of fear detected, as depicted in Table V.

TABLE V.

FOR CLASSMATES' MARKS GREATER THAN OR EQUALS TO 0,4: CORRELATION BETWEEN CLASSMATES' MARKS AND EMOTIONS $(\mathrm{N}=60)$

\begin{tabular}{|l|l|l|}
\hline Variable & r & p \\
\hline$\%$ Fear & 0,2601 & 0,05 \\
\hline
\end{tabular}

\section{CONCLUDING REMARKS AND FUTURE WORK}

Along this article we have shown an experience we have performed in order to analyze the influence of emotions on students' and instructors' marks. Thus, once introduced our objective, we have detailed our experience by exposing the goal defined, the participants involved, the environment they used, the input data and variables to analyze, the how experience itself was performed and finally the result analysis.

In this research study, we have analyzed the existing correlations between three variables associated to short 168 technical documents delivered by 100 students. For each student contribution, the three gathered variables were: its social acceptation (related to the assigned classmates' marks), its objective quality measure (related to the assigned instructors' marks) and iii) its emotion traces.

As presented in former sections, the results obtained indicate that, for the highest instructors' marks exists a positive correlation between the social acceptation of the documents and the emotion levels detected (most significantly, the level of joy detected).

On the other hand, fear seems to be the most significant emotion regarding classmates' extreme levels: for lowest social acceptation levels, a negative correlation seems to exist between instructors' marks and the fear level, whereas a positive correlation is given for classmates' marks and the level of fear detected in the documents if classmates' marks are above the average.

Based on the correlation results, we could conclude that joy is an important emotion, which should be taken into account for instructors' highest marks. Joy level seems not to be determinant for low ranked students' contributions, but seems to be significant in order to assign the highest 
instructors' mark, associated to the quality measure of the documents.

On the other hand, the fear level, as detected in the documents, seems to be significant, both for instructors (negatively, in case of low social acceptation) and classmates (positively, in case of marks above their own social acceptation rates).

Regarding the possible conclusions that could be derived from the previous results, it is worth mentioning that the concept of correlation is particularly noteworthy for the potential confusion it can cause (correlation does not imply causation). So, even correlations are found, the correlation phenomena could be caused by a third, previously unconsidered phenomenon, and there is no way to immediately infer the existence of a causal relationship between the variables considered in the research study. In that sense, we are interested in analyzing other possible variables involved, planning forthcoming research studies focused in the affective evaluation of the students' documents, and its relation with the perceived quality of them.

\section{REFERENCES}

[1] Beard C. Student Achievement: The Role of Emotions in Motivation to Learn - Emotional Maps. Pedagogic Research Project Report. Sheffield Hallam University. January 2005.

[2] Suzuki S.; Shiraishi A. and Suzuki H. An Emotional Document Investigation Tool for Academic Writing. In Proceedings of the Workhop on Research Goals and Strategies for Studying User Experience and Emotion. Lund, Sweden, 2008.

[3] Feidakis M.; Daradoumis T. and Caballé S., "Emotion Measurement in Intelligent Tutoring Systems: What, When and How to Measure," Proc. 2011 International Conference on Intelligent Networking and Collaborative Systems, IEEE Computer Society, Dec 2011, pp. 807-812, http://dx.doi.org/10.1109/INCoS.2011.82

[4] Rotaru M. and Litman D. "Using Word-level Pitch Features to Better Predict Student Emotions during Spoken Tutoring Dialogues," Proceedings of Interspeech, 2005.

[5] Nicholas, G.; Rotaru, M. and Litman, D.J.; "Exploiting Wordlevel Features for Emotion Prediction," Spoken Language Technology Workshop 2006. IEEE, 2006, pp.110-113, 2006 http://dx.doi.org/10.1109/SLT.2006.326829

[6] Xiaoqing Liu; Lei Zhang; Yadegar, J. and Kamat, N.; "A Robust Multi-Modal Emotion Recognition Framework for Intelligent Tutoring Systems," IEEE International Conference on Advanced Learning Technologies (ICALT) 2011, pp.63-65, http://dx.doi.org/10.1109/ICALT.2011.26

[7] Feldman, R., Techniques and Applications for Sentiment Analysis, Communications of the ACM Vol. 56, 2013, pp. 82-89. http://dx.doi.org/10.1145/2436256.2436274

[8] Liu, B., Sentiment Analysis and Subjectivity, Indurkhya, N., Damerau, F.J. (eds.) Handbook of Natural Language Processing, 2 edn. 2010

[9] Strappavana C. and Mihalcea R., "Learning to identify emotions in text," Proc. 23th ACM Symposium on Applied Computing (SAC'08), ACM Press, 2008, pp. 1556-1560, http://dx.doi.org/10.1145/1363686.1364052

[10] Feng Tian; Qinghua Zheng; Ruomeng Zhao; Tonghao Chen; Xinyan Jia. "Can e-Learner's emotion be recognized from interactive Chinese texts?" 13th International Conference on Computer Supported Cooperative Work in Design, 2009. CSCWD 2009, pp. 546-551. http://dx.doi.org/10.1109/CSCWD.2009.4968116

[11] Tian, Feng; Zheng, Qinghua; Zheng, Deli. "Mining patterns of eLearner emotion communication in turn level of Chinese interactive texts: Experiments and findings". 14th International Conference on Computer Supported Cooperative Work in Design, 2010 , pp. 664-670. http://dx.doi.org/10.1109/CSCWD.2010.5471892

[12] Rodriguez, P.; Ortigosa, A.; Carro, R.M. "Extracting Emotions from Texts in E-Learning Environments" Sixth International Conference on Complex, Intelligent and Software Intensive Systems
(CISIS), 2012， pp.887-892， http://dx.doi.org/10.1109/CISIS. $\underline{2012.192}$

[13] Carro, R.M., Rodríguez, P., Ortigosa, A. "Detecting and Making Use of Emotions to Enhance Student Motivation in E-learning Environments". International Journal of Continuing Engineering Education and Life-Long Learning. In press

[14] Alamán, X. and Cobos, R., KnowCat: a Web Application for Knowledge Organization, in P. P. Chen et al. (Eds.), Lecture Notes in Computer Science 1727. Springer, New York, 1999, pp. 348-359.

[15] Cobos, R. and Pifarré, M. 'Collaborative knowledge construction in the web supported by the KnowCat system', Computers \& Education vol. 50, 2008, pp. 962-978. http://dx.doi.org/10.1016/ j.compedu.2006.09.009

[16] Cobos, R., Knowledge Crystallisation supported by the KnowCat system. In: Knowledge Management / Book 3, Huei-Tse Hou (Ed.). Intech Open Access Publisher. ISBN 979-953-307-744-3. 2012, pp. 185-200.

[17] Barros, L., Rodriguez, P., and Ortigosa, A. Emotion Recognition in Texts for User Model Augmenting. In Proceedings of the XII International Conference Human-Machine Interaction (Interaccion 2012). Elche, Spain, 2012..

[18] Zinck, A. and Newen, A. "Classifying emotion: a developmental account." Synthese, vol 161:1, 2008, pp. 1-25, http://dx.doi.org/10.1007/s11229-006-9149-2

\section{AUTHORS}

Ruth Cobos is Associate Professor at the Computer Engineering Department at the Universidad Autónoma de Madrid, member of the GHIA research group. She received her $\mathrm{PhD}$ degree with honors in Computer Science from the Universidad Autónoma de Madrid, Spain, in 2003. She has been a post-doctoral researcher at Technical University Munich, Germany. She has participated in several research projects related to collaborative systems, distributed, collaborative knowledge management and elearning. During 2008 and 2009 she coordinated a project funded by the AECID, which involves researchers from Colombia and Spain. Currently, her main areas of research include social media, social learning, blended learning, MOOCs, and educational technologies. (e-mail: ruth.cobos@uam.es)

Francisco Jurado is Lecturer in the Computer Engineering Department at the Universidad Autónoma de Madrid, Spain. He received his Ph.D. degree with honors in Computer Science from the University of Castilla-La Mancha in 2010. His research areas include Intelligent Tutoring Systems, Heterogeneous Distributed eLearning Systems, eLearning Standards and Computer Supported Collaborative Environments. (francisco.jurado@uam.es).

Alvaro Ortigosa is Associate Professor in the Computer Engineering Department at the Universidad Autónoma de Madrid, member of the GHIA research group and the Forensics and Security Research Institute (ICFS). He received his $\mathrm{Ph} . \mathrm{D}$. degree in Computer Science from the Universidad Autónoma de Madrid. He has worked in software engineering support environments, software reuse, adaptive systems, collaborative systems, user modeling, mobile environments, and the authoring and evaluation of adaptive systems. Currently his research interests include sentiment analysis, social network analysis, risk analysis and Cybersecurity. He is director of the Spanish Centre of Excellence on Cybersecurity and the Agency for Cybersecurity Certifications. (alvaro.ortigosa@uam.es).

Pilar Rodriguez is Associate Professor in the Computer Engineering Department of the Universidad Autónoma de Madrid. She received her Ph.D. degree in in 1990, with 
a thesis on Computational Linguistics. She joined IBM in 1985, working at the IBM-UAM Scientific Center until 1989, when she was assigned to the Instituto de Ingeniería del Conocimiento, IIC. In November 1996 she joined UAM. At present, she is a member of the GHIA group at the UAM. Main research focuses on adaptive systems, especially for learning purposes, both in individual and collaborative environments. (pilar.rodriguez@uam.es).

This article is an extended and modified version of a paper presented at the Third International Workshop on Adaptive Learning via Interactive, Collaborative and Emotional approaches (ALICE 2013), held on July 3-5, 2013, in Taichung, Taiwan, in conjunction with the 7th International Conference on Complex, Intelligent and Software Intensive Systems (CISIS 2013).This research was partially funded by the Spanish National Plan of $\mathrm{R}+\mathrm{D}$, project numbers TIN2010-17344, TIN201124139 and TIN2011-29542-C02-02, and by the Autonomous Community of Madrid, e-Madrid project, number S2009/TIC-1650. Submitted 24 March 2014. Published as re-submitted by the authors 27 April 2014. 